\title{
Data report: cultivation of prokaryotes from subsurface marine sediments of the Baltic Sea (IODP Expedition 347) ${ }^{1}$
}

\author{
Martin Krüger ${ }^{2}$ and Axel Schippers ${ }^{2}$
}

\begin{abstract}
Chapter contents
Abstract .................. 1

Introduction $\ldots \ldots \ldots \ldots \ldots \ldots \ldots \ldots$

Materials and methods............

Results................... 2

Acknowledgments............... 3

References................... 3

Tables................... 4
\end{abstract}

${ }^{1}$ Krüger, M., and Schippers, A., 2017. Data report: cultivation of prokaryotes from subsurface marine sediments of the Baltic Sea (IODP Expedition 347) In Andrén, T., Jørgensen, B.B., Cotterill, C., Green, S., and the Expedition 347 Scientists, Proceedings of the Integrated Ocean Drilling Program, 347:

College Station, TX (Integrated Ocean Drilling Program).

doi:10.2204/iodp.proc.347.201.2017

${ }^{2}$ Federal Institute for Geosciences and Natural Resources (BGR), Stilleweg 2, 30655 Hannover, Germany. Correspondence author: martin.krueger@bgr.de

\section{Abstract}

Integrated Ocean Drilling Program (IODP) Expedition 347 to the Baltic Sea in 2013 was in line with the IODP Science Plan main research theme "Deep biosphere responses to glacial-interglacial cycles," addressing questions such as deep biosphere evolution, its biogeochemical processes, and how the postglacial diffusive penetration of conservative seawater ions may alter the chemical composition and microbial physiology in the subseafloor biosphere. Consequently, we tried to enrich indigenous microorganisms at in situ conditions using a broad range of electron acceptors (for fermenters; Fe, Mn, and sulfate reducers; and methanogens), simple and complex carbon substrates (in mixtures or as single compounds), and a wide range of culture conditions (temperature and salinity) to cover varying environmental conditions and metabolic requirements. The most successful were enrichment cultures with a mix of polymeric substrates, which proved to be successful for all samples investigated. Also, iron- and manganese-reducing organisms could be enriched from all sites, whereas nitrate as an electron acceptor did not work well. Methanogenic enrichments were only successful for a few of the samples investigated. In these cases, different monomeric as well as complex substrates were converted to methane, indicating a metabolically versatile indigenous microbial community in the sediments.

\section{Introduction}

Cultivation is the only way to obtain microorganisms and study their physiology in order to estimate their impact on biogeochemical cycles in deep sediments. For this reason, we enriched at in situ temperature and salinity various types of heterotrophic microorganisms, in particular fermenters; Fe(III), Mn(IV), and nitrate reducers; and methanogens, using different media that cover a wide range of environmental conditions and metabolic requirements.

Since methanogens only convert small compounds into methane, the initially more complex compounds have to be broken down by networks of sometimes syntrophic microbial consortia. Consequently, methanogens were cultivated with a broad range of complex substrates, especially hydrocarbons, fatty acids, polymers, or algal cell fragments. 


\section{Materials and methods}

Sediment cores from four sites (M0059, M0060, M0063, and M0065) collected during Integrated Ocean Drilling Program Expedition 347 were sampled for microbiology. To avoid contamination, samples for were only taken from the center of each sediment core using sterilized cut $5 \mathrm{~mL}$ syringes. Depth intervals for sampling were selected based on the quality of the cores with a higher depth resolution near the surface and a lower one at greater depth. The samples were put in anaerobic bags and sent to the Federal Institute for Geosciences and Natural Resources (BGR; Germany) at $2^{\circ}-8^{\circ} \mathrm{C}$ and stored at $8^{\circ} \mathrm{C}$ until use for enrichments.

The media for enrichment of anaerobic, marine deep biosphere organisms were based on artificial seawater as described by Batzke et al. (2007). The salinity of the medium was adjusted by dilution with water to $1 \%$, $5 \%$, $10 \%$, $12 \%$, 20\%, $25 \%$, and $30 \%$ o $\mathrm{NaCl}$. For heterotrophic fermenting organisms, the media were amended with a polymer or monomer solution. The polymer solution contained chitin, cellulose, and peptone at $0.5 \mathrm{~g} / \mathrm{L}$ each, whereas the monomer solution contained 36 different carbon sources like amino acids, fatty acids, organic acids, alcohols, and glucose (total final concentration of added organic carbon was $0.1 \mathrm{mM}$ ). HEPES buffer (4[2-hydroxyetheyl]-1-piperazineethanesulfonic acid; $2.38 \mathrm{~g} / \mathrm{L}$ ) was added and the $\mathrm{pH}$ was adjusted to 7.27.4 by addition of $\mathrm{NaOH}$ before autoclaving. After autoclaving, the medium was cooled under $\mathrm{N}_{2}$ flow, and a solution of 10 vitamins (Balch et al., 1979) and sodium bicarbonate $(0.2 \mathrm{~g} / \mathrm{L})$ was added from sterile stock solutions. For the enrichment of nitrate-, iron-, or manganese-reducing bacteria, $\mathrm{KNO}_{3}$, solid ferrihydrite, or manganese oxide, respectively, were added to reach final concentrations of $2 \mathrm{mM}$ for nitrate and $10 \mathrm{mM}$ for $\mathrm{Mn}$ and Fe. To obtain quantitative information for particular physiological groups, the most probable number (MPN) method with three parallel tubes for each 10-fold dilution was applied (Hungate, 1968). The enrichment cultures were incubated at $4^{\circ} \mathrm{C}$ for about 10 months. Growth of all cultures was checked by visual inspection (turbidity) and phase contrast microscopy. Tubes with ferrihydrite as the electron acceptor were counted positive when Fe(II) was higher than $5 \mathrm{mM}$, and tubes with manganese oxide as the electron acceptor were counted positive when decolorization of the black manganese mineral was clearly visible by eye. Tubes for testing nitrate-dependent $\mathrm{Fe}$ (II)-oxidation conditions were also set up and counted as positive when a clear precipitation of Fe(III) was visible.
To selectively enrich methanogens, autoclaved Hungate tubes were filled with $7 \mathrm{~mL}$ of sterile anoxic sulfate-free minimal medium (Widdel and Bak, 1992). Sediment slurries from each sample (1:1 mix of sediment and anaerobic medium) were anaerobically prepared, and $3 \mathrm{~mL}$ was added to the Hungate tubes. Then the tubes were purged with dinitrogen (100\%) and sealed with sterile butyl rubber stoppers and screw caps. Anaerobic enrichments were set up under methanogenic conditions without the addition of electron acceptors in triplicate and incubated vertically in the dark at $8^{\circ} \mathrm{C}$ with or without additional carbon sources. As additional carbon sources, combinations of typical sedimentary substrates were added as monomer or polymer mix (Batzke et al., 2007), Spirulina extract (all: $50 \mu \mathrm{L}$ of a $0.005 \%$ stock solution), or single compounds (betaine, palmitate, phytane, or pristane [ $25 \mu \mathrm{L}$ of a $2 \%$ stock solution]). Substrate concentrations were kept low to resemble conditions in deep sediments. In addition to these more complex substrates, simple well-known substrates for methanogens were used (acetate [0.5 mM], methanol $[0.1 \mathrm{mM}]$, trimethylamine [TMA] [0.5 $\mathrm{mM}]$, and hydrogen $\left.\left[\mathrm{H}_{2} / \mathrm{CO}_{2} / \mathrm{N}_{2} 5 \% / 5 \% / 90 \%\right]\right)$. Furthermore, the potential for hydrocarbon degradation was studied in incubations with hexadecane $(10 \mu \mathrm{L})$, ethylbenzene, dotriacontan $\left(\mathrm{C}_{32}\right)$, or methylnaphthalene $(20 \mu \mathrm{L}$ of a $2 \%$ stock solution $[\mathrm{v} / \mathrm{v}])$, which were all added directly to the medium. The increase of methane in the headspace was measured monthly over a 12 month period as previously described (Krüger et al., 2002). Positive enrichment cultures had substantially higher methane concentrations in the headspace than not inoculated control tubes.

\section{Results}

Heterotrophic enrichments were obtained for samples from all four sites (M0059, M0060, M0063, and M0065) and up to depths of 120 meters below seafloor (mbsf) (Table T1). Heterotrophic enrichments using a polymer mix obtained the highest positive dilution steps $\left(10^{-4}\right)$, whereas with a monomer mix the MPN values never exceeded $10^{-1}$. Iron- and manganese-reducing organisms could be enriched from all sites at neutral $\mathrm{pH}$ (7.5) and different salt concentrations $(1 \% 0-30 \% 0)$. As a general observation, iron and manganese reduction started earlier in the test tubes at salinity $\leq 10 \%$ o than in the test tubes with higher salinity. This was not observed under nitratereducing or heterotrophic conditions. For nitrate-dependent Fe(II)-oxidation conditions, the MPN value was never higher than $10^{-1}$, and positive enrich- 
ments were obtained only from cores from Sites M0060 and M0065.

Generally, no or only very low methane production was observed in the incubations without the addition of substrates, indicating that substrate availability for the indigenous methanogenic microbial communities was low in all samples (Tables T2 and T3). The only exceptions were one sample from Site M0059 (Sample 347-M0059E-13H-2, 125-130 cm) and two samples from Site M0063 (Samples 347M0063E-3H-2, 60-65 cm, and 12H-2, 62-67 cm), where methane in the headspace continuously increased over the 12 months of incubation time.

These three samples were also most prominently stimulated by the addition of different substrates, both with simple, monomeric, or complex compounds. Besides well-known substrates for methanogens like acetate, methanol, hydrogen, or TMA (Table T2), more complex compounds from the polymer mix, palmitate, phytane, or hydrocarbons were also degraded. This indicates the presence of a metabolically diverse active community in the sediments, enabling the breakdown of the larger compounds to smaller substrates for the methanogenic organisms at the end of the food chain.

In other sediment samples, only in a few single cases was stimulation of methane production observed. Here, the traditional methanogenic substrates and monomeric compounds seemed to be more favorable, as one would expect. The hydrocarbons tested in the enrichment cultures were the least successful substrates tested, with only one positive sediment sample (347-M0063E-12H-2, 62-67 cm).

\section{Acknowledgments}

This research used samples and/or data provided by the Integrated Ocean Drilling Program (IODP).

\section{References}

Balch, W.E., Fox, G.E., Magrum, L.J., Woese, C.R., and Wolfe, R.S., 1979. Methanogens: reevaluation of a unique biological group. Microbiological Reviews, 43(2):260-296. http://mmbr.asm.org/content/43/2/ 260.full.pdf + html

Batzke, A., Engelen, B., Sass, H., and Cypionka, H., 2007. Phylogenetic and physiological diversity of cultured deep-biosphere bacteria from equatorial Pacific Ocean and Peru margin sediments. Geomicrobiology Journal, 24(3-4):261-273. http://dx.doi.org/10.1080/ 01490450701456453

Hungate, R.E., 1968. A roll tube method for cultivation of strict anaerobes. Methods in Microbiology, 3(B):117-132. http://dx.doi.org/10.1016/S0580-9517(08)70503-8

Krüger, M., Eller, G., Conrad, R., and Frenzel, P., 2002. Seasonal variation in pathways of $\mathrm{CH}_{4}$ production and in $\mathrm{CH}_{4}$ oxidation in rice fields determined by stable carbon isotopes and specific inhibitors. Global Change Biology, 8(3):265-280. http://dx.doi.org/10.1046/j.13652486.2002.00476.x

Widdel, F., and Bak, F., 1992. Gram-negative mesophilic sulfate-reducing bacteria. In Balows, E., Trüper, H.G., Dworkin, M., Harder, W., and Schleifer, K.-H. (Eds.), The Prokaryotes (2nd edition): New York (Springer-Verlag), 3352-3378. http://dx.doi.org/10.1007/978-1-47572191-1_21

Initial receipt: 20 October 2016

Acceptance: 22 February 2017

Publication: 25 April 2017

MS 347-201 
Table T1. Results for most probable number enrichment cultures with different media incubated close to in situ temperature of $8^{\circ} \mathrm{C}$ under anaerobic conditions at different salinities, Expedition 347.

\begin{tabular}{|c|c|c|c|c|c|c|c|c|}
\hline $\begin{array}{l}\text { Core, } \\
\text { section }\end{array}$ & $\begin{array}{l}\text { Depth } \\
\text { (mbsf) }\end{array}$ & $\begin{array}{c}\text { Salinity } \\
(\% \circ)\end{array}$ & $\mathrm{Fe}(\mathrm{OH})_{3}$ & $\mathrm{MnOOH}$ & $\mathrm{KNO}_{3}$ & $\mathrm{KNO}_{3}+\mathrm{Fe}(\mathrm{II})$ & Polymer & Monomer \\
\hline \multicolumn{9}{|c|}{ 347-M0059C- } \\
\hline $3 \mathrm{H}-2$ & 8 & 25 & $10^{-2}$ & - & $10^{-2}$ & - & $10^{-4}$ & $10^{-2}$ \\
\hline $5 \mathrm{H}-2$ & 14 & 25 & $10^{-1}$ & $10^{-1}$ & - & - & $10^{-3}$ & $10^{-1}$ \\
\hline $15 \mathrm{H}-1$ & 45 & 20 & - & - & $10^{-1}$ & - & $10^{-1}$ & $10^{-1}$ \\
\hline $21 \mathrm{H}-1$ & 64 & 10 & - & - & - & - & - & - \\
\hline $25 \mathrm{H}-1$ & 78 & 10 & - & - & - & - & $10^{-1}$ & - \\
\hline \multicolumn{9}{|c|}{ 347-M0060B- } \\
\hline $13 \mathrm{H}-2$ & 37 & 30 & $10^{-1}$ & - & $10^{-3}$ & - & $10^{-4}$ & $10^{-1}$ \\
\hline $14 \mathrm{H}-2$ & 41 & 30 & $10^{-1}$ & - & $10^{-3}$ & $10^{-1}$ & $10^{-4}$ & $10^{-1}$ \\
\hline $23 \mathrm{H}-2$ & 70 & 30 & $10^{-1}$ & - & $10^{-3}$ & $10^{-1}$ & $10^{-3}$ & $10^{-1}$ \\
\hline $27 \mathrm{H}-2$ & 82 & 30 & - & - & $10^{-1}$ & $10^{-1}$ & $10^{-3}$ & $10^{-1}$ \\
\hline $28 \mathrm{H}-2$ & 85 & 10 & $10^{-1}$ & $10^{-1}$ & $10^{-1}$ & - & $10^{-1}$ & - \\
\hline \multicolumn{9}{|c|}{ 347-M0063E- } \\
\hline $1 \mathrm{H}-2$ & 2 & 1 & $10^{-3}$ & $10^{-3}$ & $10^{-3}$ & - & $10^{-4}$ & $10^{-1}$ \\
\hline $6 \mathrm{H}-2$ & 12 & 1 & $10^{-3}$ & $10^{-3}$ & $10^{-3}$ & - & $10^{-4}$ & $10^{-1}$ \\
\hline $21 \mathrm{H}-2$ & 41 & 5 & - & - & - & - & $10^{-2}$ & $10^{-1}$ \\
\hline $27 \mathrm{H}-2$ & 55 & 1 & $10^{-3}$ & $10^{-3}$ & $10^{-3}$ & - & $10^{-4}$ & $10^{-1}$ \\
\hline $33 \mathrm{H}-2$ & 66 & 5 & - & - & - & - & $10^{-1}$ & - \\
\hline $40 \mathrm{H}-2$ & 80 & 5 & - & - & - & - & $10^{-1}$ & - \\
\hline $43 \mathrm{H}-2$ & 90 & 5 & - & - & - & - & - & - \\
\hline \multicolumn{9}{|c|}{ 347-M0065C- } \\
\hline $4 \mathrm{H}-2$ & 11 & 12 & $10^{-3}$ & $10^{-1}$ & $10^{-4}$ & $10^{-1}$ & $10^{-4}$ & $10^{-1}$ \\
\hline $7 \mathrm{H}-2$ & 20 & 12 & $10^{-3}$ & $10^{-1}$ & $10^{-3}$ & $10^{-1}$ & $10^{-4}$ & $10^{-1}$ \\
\hline $10 \mathrm{H}-2$ & 30 & 12 & - & - & $10^{-1}$ & - & $10^{-1}$ & - \\
\hline $12 \mathrm{H}-2$ & 37 & 1 & - & - & $10^{-1}$ & - & $10^{-1}$ & - \\
\hline
\end{tabular}

Ferrihydrite, manganese oxide, or $\mathrm{KNO}_{3}$ were added as the electron acceptor each for enrichment of heterotrophs. Fe(II) was added for enrichment of nitrate-reducing lithotrophs. Mixtures of organic polymers or monomers served for enrichment of heterotrophic fermenters. Numbers = positive test tubes with the highest dilution step. $-=$ no successful enrichment. 
Table T2. Results of methanogenic enrichment cultures by substrate after 12 months of incubation time, Expedition 347.

\begin{tabular}{|c|c|c|c|c|c|c|c|c|c|}
\hline $\begin{array}{l}\text { Core, section, } \\
\text { interval }(\mathrm{cm})\end{array}$ & No substrate & Acetate & $\mathrm{H}_{2} / \mathrm{CO}_{2}$ & Methanol & TMA & Palmitate & Betaine & Phytane & Pristane \\
\hline \multicolumn{10}{|l|}{ 347-M0059C- } \\
\hline $3 \mathrm{H}-2,40-45$ & $(+)$ & - & - & - & - & - & - & - & - \\
\hline $6 \mathrm{H}-1,95-100$ & $(+)$ & - & - & - & - & - & - & - & - \\
\hline $9 \mathrm{H}-1,95-100$ & $(+)$ & - & - & - & + & - & - & - & - \\
\hline $12 \mathrm{H}-2,65-70$ & $(+)$ & - & - & - & - & - & - & - & - \\
\hline $15 \mathrm{H}-1,100-105$ & $(+)$ & - & $(+)$ & - & + & - & - & - & - \\
\hline $24 \mathrm{H}-2,65-70$ & - & - & - & - & - & - & - & - & - \\
\hline \multicolumn{10}{|l|}{ 347-M0059E- } \\
\hline $4 \mathrm{H}-2,55-60$ & - & - & - & - & - & - & - & - & - \\
\hline 7H-2, 115-120 & - & - & - & - & - & - & - & - & - \\
\hline $10 \mathrm{H}-2,140-145$ & - & - & - & - & - & - & - & - & - \\
\hline $13 \mathrm{H}-2,125-130$ & + & + & ++ & ++ & + & + & + & + & + \\
\hline $16 \mathrm{H}-2,90-95$ & - & - & - & - & - & - & - & - & - \\
\hline $25 \mathrm{H}-2,105-110$ & - & - & - & - & + & - & - & - & - \\
\hline \multicolumn{10}{|l|}{ 347-M0060B- } \\
\hline $6 \mathrm{H}-2,75-80$ & - & - & - & - & - & - & - & - & - \\
\hline $9 \mathrm{H}-2,55-60$ & - & - & - & - & - & - & - & - & - \\
\hline $12 \mathrm{H}-2,50-55$ & - & - & - & - & - & - & - & - & - \\
\hline $15 \mathrm{H}-2,45-50$ & - & - & - & - & - & - & - & - & - \\
\hline $23 \mathrm{H}-2,20-25$ & - & - & - & - & - & - & - & - & - \\
\hline $24 \mathrm{H}-2,50-55$ & - & - & - & - & - & - & - & - & - \\
\hline $28 \mathrm{H}-2,110-115$ & - & - & - & - & - & - & - & - & - \\
\hline \multicolumn{10}{|l|}{ 347-M0063E- } \\
\hline $3 \mathrm{H}-2,60-65$ & + & + & ++ & $(+)$ & ++ & - & + & - & - \\
\hline $6 \mathrm{H}-2,50-55$ & $(+)$ & - & - & + & - & - & - & - & - \\
\hline $9 \mathrm{H}-2,45-50$ & - & - & - & - & - & - & - & - & - \\
\hline $12 \mathrm{H}-2,62-67$ & + & + & ++ & ++ & + & + & + & + & + \\
\hline $15 \mathrm{H}-2,45-50$ & - & - & - & - & - & - & - & - & - \\
\hline $18 \mathrm{H}-2,25-30$ & - & - & - & - & - & - & - & - & - \\
\hline $21 \mathrm{H}-2,25-30$ & $(+)$ & - & - & + & - & $(+)$ & $(+)$ & $(+)$ & - \\
\hline $32 \mathrm{H}-2,90-95$ & - & - & - & - & - & - & - & - & - \\
\hline $42 \mathrm{H}-2,120-125$ & - & - & - & - & - & - & - & - & - \\
\hline \multicolumn{10}{|l|}{ 347-M0065C- } \\
\hline $4 \mathrm{H}-2,60-65$ & - & - & - & - & - & - & - & - & - \\
\hline $7 \mathrm{H}-2,65-70$ & - & - & - & - & - & - & - & - & - \\
\hline $10 \mathrm{H}-2,60-65$ & - & - & - & - & - & - & - & - & - \\
\hline $12 \mathrm{H}-2,110-115$ & - & - & - & - & - & - & - & - & - \\
\hline
\end{tabular}

TMA = trimethylamine. $(+)=\mathrm{CH}_{4}$ production only slightly higher than in controls, $+=\mathrm{CH}_{4}$ formation substantially higher than in controls, $++=$ strong and long-lasting $\mathrm{CH}_{4}$ formation activity, $-=$ no $\mathrm{CH}_{4}$ formation. 
Table T3. Results of methanogenic enrichment cultures with complex or hydrocarbon substrates after 12 months incubation, Expedition 347.

\begin{tabular}{|c|c|c|c|c|c|c|c|c|}
\hline $\begin{array}{l}\text { Core, section, } \\
\text { interval }(\mathrm{cm})\end{array}$ & $\begin{array}{c}\text { No } \\
\text { substrate }\end{array}$ & $\begin{array}{l}\text { Monomer } \\
\text { mix }\end{array}$ & $\begin{array}{l}\text { Polymer } \\
\text { mix }\end{array}$ & Spirulina & Hexadecane & Ethylbenzene & Methylnaphthalene & Dotriacontan \\
\hline \multicolumn{9}{|l|}{ 347-M0059C- } \\
\hline $3 \mathrm{H}-2,40-45$ & $(+)$ & - & - & - & - & - & - & - \\
\hline $6 \mathrm{H}-1,95-100$ & $(+)$ & - & - & - & - & - & - & - \\
\hline $9 \mathrm{H}-1,95-100$ & $(+)$ & - & - & - & - & - & - & - \\
\hline $12 \mathrm{H}-2,65-70$ & $(+)$ & - & - & - & - & - & - & - \\
\hline $15 \mathrm{H}-1,100-105$ & $(+)$ & - & - & - & - & - & - & - \\
\hline $24 \mathrm{H}-2,65-70$ & - & - & - & - & - & - & - & - \\
\hline \multicolumn{9}{|l|}{ 347-M0059E- } \\
\hline $4 \mathrm{H}-2,55-60$ & - & - & - & - & - & - & - & - \\
\hline 7H-2, 115-120 & - & - & - & - & - & - & - & - \\
\hline $10 \mathrm{H}-2,140-145$ & - & - & - & + & & - & - & - \\
\hline $13 \mathrm{H}-2,125-130$ & + & ++ & + & + & $(+)$ & - & - & - \\
\hline $16 \mathrm{H}-2,90-95$ & - & - & - & - & - & - & - & - \\
\hline $25 \mathrm{H}-2,105-110$ & - & - & - & - & - & - & - & - \\
\hline \multicolumn{9}{|l|}{ 347-М0060B- } \\
\hline $6 \mathrm{H}-2,75-80$ & - & - & - & - & - & - & - & - \\
\hline $9 \mathrm{H}-2,55-60$ & - & - & - & - & - & - & - & - \\
\hline $12 \mathrm{H}-2,50-55$ & - & - & - & - & - & - & - & - \\
\hline $15 \mathrm{H}-2,45-50$ & - & - & - & - & - & - & - & - \\
\hline $23 \mathrm{H}-2,20-25$ & - & - & - & - & - & - & - & - \\
\hline $24 \mathrm{H}-2,50-55$ & - & - & - & - & - & - & - & - \\
\hline $28 \mathrm{H}-2,110-115$ & - & - & - & - & - & - & - & - \\
\hline \multicolumn{9}{|l|}{ 347-M0063E- } \\
\hline $3 \mathrm{H}-2,60-65$ & + & - & - & + & - & - & - & - \\
\hline $6 \mathrm{H}-2,50-55$ & $(+)$ & - & - & - & - & - & - & - \\
\hline $9 \mathrm{H}-2,45-50$ & - & - & - & - & - & - & - & - \\
\hline $12 \mathrm{H}-2,62-67$ & + & + & + & + & + & + & + & $(+)$ \\
\hline $15 \mathrm{H}-2,45-50$ & - & - & - & - & - & - & - & - \\
\hline $18 \mathrm{H}-2,25-30$ & - & - & - & - & - & - & - & - \\
\hline $21 \mathrm{H}-2,25-30$ & $(+)$ & $(+)$ & $(+)$ & $(+)$ & $(+)$ & - & - & - \\
\hline $32 \mathrm{H}-2,90-95$ & - & - & - & - & - & - & - & - \\
\hline $42 \mathrm{H}-2,120-125$ & - & - & - & - & - & - & - & - \\
\hline \multicolumn{9}{|l|}{ 347-M0065C- } \\
\hline $4 \mathrm{H}-2,60-65$ & - & - & - & - & - & - & - & - \\
\hline $7 \mathrm{H}-2,65-70$ & - & - & - & - & - & - & - & - \\
\hline $10 \mathrm{H}-2,60-65$ & - & - & - & - & - & - & - & - \\
\hline $12 \mathrm{H}-2,110-115$ & - & - & - & - & - & - & - & - \\
\hline
\end{tabular}

$(+)=\mathrm{CH}_{4}$ production only slightly higher than in controls, $+=\mathrm{CH}_{4}$ formation substantially higher than in controls, $++=$ strong and long-lasting $\mathrm{CH}_{4}$ formation activity, $-=$ no $\mathrm{CH}_{4}$ formation. 